\title{
Methods with discrete sources for electromagnetic scattering by large axisymmetric particles with extreme geometries ${ }^{\text {th }}$
}

\author{
Adrian Doicu ${ }^{\mathrm{a}}$, Yuri Eremin ${ }^{\mathrm{b}}$, Dmitry S. Efremenko ${ }^{\mathrm{a}}$, Thomas Trautmann ${ }^{\mathrm{a}}$ \\ ${ }^{a}$ Remote Sensing Technology Institute, German Aerospace Centre, Oberpfaffenhofen, Wessling, Germany \\ ${ }^{b}$ Lomonosov Moscow State University, Lenin's Hills, Moscow, Russia
}

\begin{abstract}
The applicability of the discrete sources method and the null-field method with discrete sources to electromagnetic scattering by large axisymmetric particles with extreme geometries is analyzed. We present the numerical and theoretical improvements of the methods and design the null-field method with multiple spherical vector wave functions as an enhancement of the conventional null-field method for computing the optical properties of large size parameter particles. The numerical performances of the methods with discrete sources are illustrated through simulations in spheroidal and cylindrical particle cases.
\end{abstract}

\section{Introduction}

In atmospheric radiative transfer and remote sensing, accurate computations of electromagnetic scattering by large particles with extreme geometries are often required.

A first method which serves this purpose is the discrete sources method. The approximate solution to the scattering problem is constructed as a finite linear combination of fields of elementary sources. The discrete sources are placed on a certain support, and the unknown amplitudes of the discrete sources are determined from the boundary conditions. As discrete sources localized, distributed and multiple spherical vector wave functions, magnetic and electric dipoles, and systems of vector Mie potentials have been considered in [1]. Distributed spherical vector wave functions (lowest-order multipoles) have been used by Eremin and Sveshnikov [2] for analyzing the scattering by axisymmetric particles, while multiple spherical vector wave functions (multiple multipoles) have been introduced by Hafner [3] in the framework of the multiple multipole method. Eremin [4] has shown that for oblate axisymmetric particles, the use of lowest-order multipoles with origins located in the complex plane still decouples the scattering problem over the azimuthal modes and increases the stability of the computational scheme.

The null-field method (otherwise known as the extended boundary condition method) is also suitable for analizing the electromagnetic scattering by large and highly aspherical particles. The method was initially proposed by Watermann [5, 6] and later developed by Barber and Hill [7], and Mishchenko et al. [8]. In the framework of the null-field method, the transition matrix relating the expansion coefficients of the scattered and incident field is derived as

$$
\mathbf{T}=-\mathbf{Q}^{11}\left(\mathbf{Q}^{31}\right)^{-1}
$$

where the matrix $\mathbf{Q}^{31}$ is obtained by imposing the null-field condition inside a spherical surface enclosed in the particle, and the matrix $\mathbf{Q}^{11}$ is obtained from Huygens principle. The elements of the $\mathbf{Q}$ matrices are expressed as integrals of products of spherical vector wave functions over the particle surface. Despite its wide range of applicability, the method suffers from numerical problems regarding convergence and loss of accuracy. The reason is that for large and/or strongly deformed particles, the matrix $\mathbf{Q}^{31}$ becomes severely

\footnotetext{
This paper has been published in JQSRT. Please, cite: Doicu et al. // JQSRT 164 (2015) $137-146$. doi:10.1016/j.jqsrt.2015.06.007
} 
ill conditioned, and the inversion process is negatively influenced by round-off errors. Essentially, small numerical errors in the computed elements of the matrix $\mathbf{Q}^{31}$ are dramatically amplified in the elements of the inverse matrix. A number of methods have been proposed to improve the numerical stability in computations for large particles with extreme geometries. We distinguish the following categories of methods:

1. Methods dealing with the numerical stability of the inversion process. The orthogonalization approach which exploits the unitarity property of the $\mathbf{T}$ matrix [6,9], the Gauss elimination method with backsubstitution [10], the block matrix inversion method [11], and the perturbation approach for the Q-matrix inversion [12] fall into this category.

2. Methods dealing with an accurate computation of the Q-matrix elements. Somerville et al. [13] found that in the case of spheroids, the numerical computation of the integrals of the $\mathbf{Q}^{31}$-matrix elements may suffer a significant loss of precision due to exact cancellations of large parts of the integrand. The sources of this problematic behaviour are some particular terms in the Laurent series expansion of the integrand. Later on, Somerville et al. [14] reformulated the integrals such that these problematic terms are removed, and designed a numerically stable implementation of the null-field method for T-matrix calculation. In the framework of the null-field method, Petrov et al. [15] developed the so called shape matrix (or Sh-matrix) approach to analyze different particle morphologies. The Q-matrix elements are expressed through analytical relations in terms of the Sh-matrix elements, which depend only on the particle shape. On the other hand, the Sh-matrix elements are determined analytically for many types of particles [16-19], and the resulting analytical solutions speed up the calculations and make them more stable.

3. Methods dealing with an accurate computation and inversion of the matrix $\mathbf{Q}^{31}$ by using extendedand the multiple-precision floating-point variables [13, 20].

4. Methods relying on formal modifications of the single spherical coordinate-based null-field method. These methods leading to better conditioned systems of equations include the iterative version of the null-field method [21, 22], and the application of sub-boundary bases for surface fields approximation [23], the spheroidal coordinate formalism [24, 25], and discrete sources [1, 26].

In the null-field method with discrete sources, a set of integral equations for the surface current densities is derived in a variety of discrete sources, while the surface current densities are approximated by fields of discrete sources. The fundamentals of the method using various types of discrete sources (essentially the same as in the discrete sources method) have been presented in [1]. In [26] and [27] only distributed spherical vector wave functions with origins located on the axis of symmetry of the particle or in the complex plane have been considered, and convergent results for prolate axisymmetric particles with a size parameter of about 100, and oblate axisymmetric particles with a size parameter of about 30 have been reported. In the present work we will pay attention to the system of multiple spherical vector wave functions with origins located in the complex plane. Although multiple spherical vector wave functions have been theoretically introduced in [1], they have not been used in [26]. By reconsidering this system of vector functions we will be able to increase the range of applicability of the null-field method to oblate particles with size parameters larger than 30 .

Our paper is organized as follows. Section 2 briefly sketches the fundamentals of the discrete sources method and the null-field method with discrete sources. As discrete sources localized, distributed and multiples spherical vector wave functions are considered. Section 3 describes the numerical and theoretical improvements of the methods for analyzing the electromagnetic scattering by large and strongly deformed axisymmetric particles. A convergence analysis for spheroidal and cylindrical particles is the objective of Section 4, while a short summary is given in Section 5 .

\section{Methods with discrete sources}

We consider a homogeneous, isotropic particle occupying a domain $D_{\mathrm{i}}$ with boundary $S$ and exterior $D_{\mathrm{s}}$. The unit normal vector to $S$ directed into $D_{\mathrm{s}}$ is denoted by $\mathbf{n}$. The exterior domain $D_{\mathrm{s}}$ is assumed to be homogeneous, isotropic and nonabsorbing. The wave number in domain $D_{\mathrm{t}}, \mathrm{t}=\mathrm{s}$, i, is $k_{\mathrm{t}}=k_{0} \sqrt{\varepsilon_{\mathrm{t}} \mu_{\mathrm{t}}}$, 
where $k_{0}$ is the wave number in the free space, and $\varepsilon_{\mathrm{t}}$ and $\mu_{\mathrm{t}}$ are the relative permittivity and permeability of the domain $D_{\mathrm{t}}$, respectively. The transmission boundary-value problem for a homogeneous and isotropic particle has the following formulation: Given $\mathbf{E}_{0}$ and $\mathbf{H}_{0}$ as an entire solution to the Maxwell equations representing the incident field, find the vector fields $\mathbf{E}_{\mathrm{s}}, \mathbf{H}_{\mathrm{s}}$ and $\mathbf{E}_{\mathrm{i}}, \mathbf{H}_{\mathrm{i}}$ satisfying the Maxwell equations

$$
\nabla \times \mathbf{E}_{\mathrm{t}}=\mathrm{j} k_{0} \mu_{\mathrm{t}} \mathbf{H}_{\mathrm{t}}, \quad \nabla \times \mathbf{H}_{\mathrm{t}}=-\mathrm{j} k_{0} \varepsilon_{\mathrm{t}} \mathbf{E}_{\mathrm{t}},
$$

in $D_{\mathrm{t}}, \mathrm{t}=\mathrm{s}$, i, the two transmission conditions

$$
\begin{aligned}
\mathbf{n} \times \mathbf{E}_{\mathrm{i}}-\mathbf{n} \times \mathbf{E}_{\mathrm{s}} & =\mathbf{n} \times \mathbf{E}_{0}, \\
\mathbf{n} \times \mathbf{H}_{\mathrm{i}}-\mathbf{n} \times \mathbf{H}_{\mathrm{s}} & =\mathbf{n} \times \mathbf{H}_{0},
\end{aligned}
$$

on $S$, and the Silver-Müller radiation condition for the scattered field

$$
\frac{\mathbf{r}}{r} \times \sqrt{\mu_{\mathbf{s}}} \mathbf{H}_{\mathbf{s}}+\sqrt{\varepsilon_{\mathbf{s}}} \mathbf{E}_{\mathbf{s}}=o\left(\frac{1}{r}\right), \text { as } r \rightarrow \infty
$$

uniformly for all directions $\mathbf{r} / r$.

Let us consider the vector functions $\boldsymbol{\Phi}_{\alpha}^{q}(k \mathbf{r})$ and $\boldsymbol{\Psi}_{\alpha}^{q}(k \mathbf{r})$, for $q=1$, 3 , with the properties (a) $\nabla \times \boldsymbol{\Phi}_{\alpha}^{q}=$ $k \boldsymbol{\Psi}_{\alpha}^{q}$ and $\nabla \times \boldsymbol{\Psi}_{\alpha}^{q}=k \boldsymbol{\Phi}_{\alpha}^{q}$, (b) $\boldsymbol{\Phi}_{\alpha}^{1}$ and $\boldsymbol{\Psi}_{\alpha}^{1}$ are finite at the origin, and (c) $\boldsymbol{\Phi}_{\alpha}^{3}$ and $\boldsymbol{\Psi}_{\alpha}^{3}$ satisfy the radiation condition. In particular, $\boldsymbol{\Phi}_{\alpha}^{q}$ and $\boldsymbol{\Psi}_{\alpha}^{q}$ stand for different types of spherical vector wave functions (SVWF), i.e.,

1. localized SVWF (localized multipoles)

$$
\boldsymbol{\Phi}_{\alpha}^{q}(k \mathbf{r})=\mathbf{M}_{m n}^{q}(k \mathbf{r}) \text { and } \mathbf{\Psi}_{\alpha}^{q}(k \mathbf{r})=\mathbf{N}_{m n}^{q}(k \mathbf{r}),
$$

where $\alpha=(m, n)$ for $m \in \mathbb{Z}$ and $n \geq \max (1,|m|)$.

2. distributed SVWF (lowest-order multipoles)

$$
\boldsymbol{\Phi}_{\alpha}^{q}(k \mathbf{r})=\mathbf{M}_{m,|m|+l}^{q}\left(k\left(\mathbf{r}-z_{n} \mathbf{e}_{\mathbf{z}}\right)\right) \text { and } \mathbf{\Psi}_{\alpha}^{q}(k \mathbf{r})=\mathbf{N}_{m,|m|+l}^{q}\left(k\left(\mathbf{r}-z_{n} \mathbf{e}_{\mathbf{z}}\right)\right),
$$

where $\left\{z_{n} \mid n \geq 1\right\}$ is a dense set of points situated on the $z$-axis, $\mathbf{e}_{\mathbf{z}}$ is the unit vector in the direction of the $z$-axis, $l=1$ if $m=0$ and $l=0$ if $m \neq 0$, and $\alpha=(m, n)$ for $m \in \mathbb{Z}$ and $n \geq 1$.

3. multiple SVWF (multiple multipoles)

$$
\boldsymbol{\Phi}_{\alpha}^{q}(k \mathbf{r})=\mathbf{M}_{m n}^{q}\left(k\left(\mathbf{r}-z_{p} \mathbf{e}_{\mathbf{z}}\right)\right) \text { and } \mathbf{\Psi}_{\alpha}^{q}(k \mathbf{r})=\mathbf{N}_{m n}^{q}\left(k\left(\mathbf{r}-z_{p} \mathbf{e}_{\mathbf{z}}\right)\right),
$$

where $\left\{z_{p} \mid p=1,2, \ldots, N_{\mathrm{p}}\right\}$ is a finite set of points (poles) situated on the $z$-axis, $N_{\mathrm{p}}$ is the number of poles, and $\alpha=(m, n)$ for $m \in \mathbb{Z}$ and $n \geq \max (1,|m|)$.

The expressions of the spherical vector wave functions with an orgin shifted at $\widehat{z}$ along the $z$-axis are given by $[26]$

$$
\begin{aligned}
\mathbf{M}_{m n}^{1,3}\left(k\left(\mathbf{r}-\widehat{z} \mathbf{e}_{\mathbf{z}}\right)\right) & =c_{n} z_{n}^{1,3}(k R)\left[\mathrm { j } m \pi _ { n } ^ { | m | } ( \widehat { \theta } ) \left(\sin (\theta-\widehat{\theta}) \mathbf{e}_{r}\right.\right. \\
& \left.\left.+\cos (\theta-\widehat{\theta}) \mathbf{e}_{\theta}\right)-\tau_{n}^{|m|}(\widehat{\theta}) \mathbf{e}_{\varphi}\right] \mathrm{e}^{\mathrm{j} m \varphi}
\end{aligned}
$$

and

$$
\begin{aligned}
\mathbf{N}_{m n}^{1,3}\left(k\left(\mathbf{r}-\widehat{z} \mathbf{e}_{z}\right)\right) & =c_{n}\left\{n(n+1) \frac{z_{n}^{1,3}(k R)}{k R} P_{n}^{|m|}(\cos \widehat{\theta})\right. \\
& \times\left(\cos (\theta-\widehat{\theta}) \mathbf{e}_{r}-\sin (\theta-\widehat{\theta}) \mathbf{e}_{\theta}\right)+\frac{\left(k R z_{n}^{1,3}(k R)\right)^{\prime}}{k R} \\
& \times\left[\tau_{n}^{|m|}(\widehat{\theta})\left(\sin (\theta-\widehat{\theta}) \mathbf{e}_{r}+\cos (\theta-\widehat{\theta}) \mathbf{e}_{\theta}\right)\right. \\
& \left.\left.+\mathrm{j} m \pi_{n}^{|m|}(\widehat{\theta}) \mathbf{e}_{\varphi}\right]\right\} \mathrm{e}^{\mathrm{j} m \varphi},
\end{aligned}
$$


where $c_{n}=1 / \sqrt{2 n(n+1)}, z_{n}^{1}$ and $z_{n}^{3}$ denote the spherical Bessel functions $j_{n}$ and the spherical Hankel functions of the first kind $h_{n}$, respectively, $P_{n}^{|m|}(\cos \theta)$ are the normalized associated Legendre functions, $\tau_{n}^{|m|}(\theta)=\mathrm{d} P_{n}^{|m|}(\cos \theta) / \mathrm{d} \theta, \pi_{n}^{|m|}(\theta)=P_{n}^{|m|}(\cos \theta) / \sin \theta$

$$
R^{2}=\rho^{2}+(z-\widehat{z})^{2}, \sin \widehat{\theta}=\frac{\rho}{R}, \cos \widehat{\theta}=\frac{z-\widehat{z}}{R}
$$

$\left(\mathbf{e}_{r}, \mathbf{e}_{\theta}, \mathbf{e}_{\varphi}\right)$ are the unit vectors in spherical coordinates, and $(r, \theta, \varphi)$ and $(\rho, \varphi, z)$ are the spherical and the cylindrical coordinates of the field point $\mathbf{r}$, respectively. The localized SVWF correspond to $\widehat{z}=0$ in (4) and (5), in which case, $R=r$ and $\widehat{\theta}=\theta$.

\subsection{Discrete sources method}

An important property of the systems of vector functions $\left\{\boldsymbol{\Phi}_{\alpha}^{q}, \boldsymbol{\Psi}_{\alpha}^{q}\right\}$ is that the sets of tangential vector functions

$$
\left\{\left(\begin{array}{c}
\mathbf{n} \times \boldsymbol{\Phi}_{\alpha}^{q_{\mathrm{t}}}\left(k_{\mathrm{t}} \mathbf{r}\right) \\
-\mathrm{j} \sqrt{\frac{\varepsilon_{\mathrm{t}}}{\mu_{\mathrm{t}}}} \mathbf{n} \times \mathbf{\Psi}_{\alpha}^{q_{\mathrm{t}}}\left(k_{\mathrm{t}} \mathbf{r}\right)
\end{array}\right),\left(\begin{array}{c}
\mathbf{n} \times \mathbf{\Psi}_{\alpha}^{q_{\mathrm{t}}}\left(k_{\mathrm{t}} \mathbf{r}\right) \\
-\mathrm{j} \sqrt{\frac{\varepsilon_{\mathrm{t}}}{\mu_{\mathrm{t}}}} \mathbf{n} \times \boldsymbol{\Phi}_{\alpha}^{q_{\mathrm{t}}}\left(k_{\mathrm{t}} \mathbf{r}\right)
\end{array}\right)\right\}
$$

are complete on the surface $S$, i.e., for any tangential field $\left(\mathbf{e}_{0}, \mathbf{h}_{0}\right)$ and any $\delta>0$, there exists $N_{0}=N_{0}(\delta)$, such that for all $N>N_{0}$,

$$
\left\|\mathbf{e}_{0}+\mathbf{e}_{\mathrm{s}}^{N}-\mathbf{e}_{\mathbf{i}}^{N}\right\|_{2 S}+\left\|\mathbf{h}_{0}+\mathbf{h}_{\mathrm{s}}^{N}-\mathbf{h}_{\mathrm{i}}^{N}\right\|_{2 S} \leq \delta
$$

where $\mathbf{e}_{0}=\mathbf{n} \times \mathbf{E}_{0}, \mathbf{h}_{0}=\mathbf{n} \times \mathbf{H}_{0}$, and

$$
\left(\begin{array}{c}
\mathbf{e}_{\mathrm{t}}^{N}(\mathbf{r}) \\
\mathbf{h}_{\mathrm{t}}^{N}(\mathbf{r})
\end{array}\right)=\sum_{\alpha=1}^{N} a_{\mathrm{t} \alpha}\left(\begin{array}{c}
\mathbf{n} \times \boldsymbol{\Phi}_{\alpha}^{q_{\mathrm{t}}}\left(k_{\mathrm{t}} \mathbf{r}\right) \\
-\mathrm{j} \sqrt{\frac{\varepsilon_{\mathrm{t}}}{\mu_{\mathrm{t}}}} \mathbf{n} \times \Psi_{\alpha}^{q_{\mathrm{t}}}\left(k_{\mathrm{t}} \mathbf{r}\right)
\end{array}\right)+b_{\mathrm{t} \alpha}\left(\begin{array}{c}
\mathbf{n} \times \mathbf{\Psi}_{\alpha}^{q_{\mathrm{t}}}\left(k_{\mathrm{t}} \mathbf{r}\right) \\
-\mathrm{j} \sqrt{\frac{\varepsilon_{\mathrm{t}}}{\mu_{\mathrm{t}}}} \mathbf{n} \times \mathbf{\Phi}_{\alpha}^{q_{\mathrm{t}}}\left(k_{\mathrm{t}} \mathbf{r}\right)
\end{array}\right)
$$

with $\mathbf{e}_{\mathrm{t}}=\mathbf{n} \times \mathbf{E}_{\mathrm{t}}, \mathbf{h}_{\mathrm{t}}=\mathbf{n} \times \mathbf{H}_{\mathrm{t}}, \mathrm{t}=\mathrm{s}, \mathrm{i}, q_{\mathrm{s}}=3$ and $q_{\mathrm{i}}=1$. As a result, the amplitudes of the discrete sources can be obtained by minimising the residual fields on the particle surface

$$
\mathbf{x}=\arg \min \left(\left\|\mathbf{e}_{0}+\mathbf{e}_{\mathrm{s}}^{N}-\mathbf{e}_{\dot{i}}^{N}\right\|_{2 S}^{2}+\left\|\mathbf{h}_{0}+\mathbf{h}_{\mathbf{s}}^{N}-\mathbf{h}_{\mathbf{i}}^{N}\right\|_{2 S}^{2}\right)
$$

where $\mathbf{x}=\left[a_{\mathrm{i} \alpha}, b_{\mathrm{i} \alpha}, a_{\mathbf{s} \alpha}, b_{\mathbf{s} \alpha}\right]^{T}$ is a $4 N$-dimensional vector. As this procedure leads to a normal system of equations which is fundamentally unstable for amplitudes determination, the point matching method is used to satisfy the boundary conditions. Choosing a set of matching points $\left\{\mathbf{r}_{p}\right\}_{p=1}^{P}, P>N$, on the particle surface, the unknown vector $\mathbf{x}$ is computed from (8) as the least squares solution $\mathbf{x}=\operatorname{argmin} \mathbf{y}\|\mathbf{A y}-\mathbf{b}\|^{2}$, where $\mathbf{A}$ is a $4 P \times 4 N$ overdetermined matrix, while the entries of the $4 P$-dimensional vector $\mathbf{b}$ are specified by the values of the incident field at the matching points.

\subsection{Null-field method with discrete sources}

In the null-field method with discrete sources, the internal surface fields $\mathbf{e}_{i}$ and $\mathbf{h}_{i}$ are approximated by (7), and the expansion coefficients $a_{\mathrm{i} \alpha}$ and $b_{\mathrm{i} \alpha}$ are determined from the null-field equations

$$
\begin{aligned}
& \frac{\mathrm{j} k_{\mathrm{s}}^{2}}{\pi} \int_{S}\left[\left(\mathbf{e}_{\mathrm{i}}^{N}(\mathbf{r})-\mathbf{e}_{0}(\mathbf{r})\right) \cdot\left(\begin{array}{c}
\mathbf{\Psi}_{\bar{\alpha}}^{3}\left(k_{\mathrm{s}} \mathbf{r}\right) \\
\boldsymbol{\Phi}_{\bar{\alpha}}^{3}\left(k_{\mathbf{s}} \mathbf{r}\right)
\end{array}\right)\right. \\
& \left.+\mathrm{j} \sqrt{\frac{\mu_{\mathrm{s}}}{\varepsilon_{\mathbf{s}}}}\left(\mathbf{h}_{\mathrm{i}}^{N}(\mathbf{r})-\mathbf{h}_{0}(\mathbf{r})\right) \cdot\left(\begin{array}{c}
\boldsymbol{\Phi}_{\bar{\alpha}}^{3}\left(k_{\mathrm{s}} \mathbf{r}\right) \\
\boldsymbol{\Psi}_{\bar{\alpha}}^{3}\left(k_{\mathrm{s}} \mathbf{r}\right)
\end{array}\right)\right] \mathrm{d} S(\mathbf{r})=0,
\end{aligned}
$$

for $\bar{\alpha}=(-m, n)$ and $\bar{\alpha}=1,2, \ldots, N$. Using the vector spherical wave expansion of the incident field,

$$
\mathbf{E}_{0}(\mathbf{r})=\sum_{\alpha=1}^{N} a_{0 \alpha} \mathbf{M}_{\alpha}^{1}\left(k_{\mathbf{s}} \mathbf{r}\right)+b_{0 \alpha} \mathbf{N}_{\alpha}^{1}\left(k_{\mathrm{s}} \mathbf{r}\right)
$$


yields

$$
\mathbf{Q}^{31}\left(k_{\mathbf{s}}, k_{\mathbf{i}}\right)\left[\begin{array}{c}
a_{\mathbf{i} \beta} \\
b_{\mathbf{i} \beta}
\end{array}\right]=-\mathbf{Q}_{0}^{31}\left(k_{\mathbf{s}}, k_{\mathbf{s}}\right)\left[\begin{array}{c}
a_{0 \beta} \\
b_{0 \beta}
\end{array}\right],
$$

where the entries of the matrix $\mathbf{Q}^{31}\left(k_{\mathbf{s}}, k_{\mathrm{i}}\right)$,

$$
\mathbf{Q}^{31}\left(k_{\mathbf{s}}, k_{\mathrm{i}}\right)=\left[\begin{array}{cc}
\left(Q^{31}\right)_{\alpha \beta}^{11} & \left(Q^{31}\right)_{\alpha \beta}^{12} \\
\left(Q^{31}\right)_{\alpha \beta}^{21} & \left(Q^{31}\right)_{\alpha \beta}^{22}
\end{array}\right]
$$

are given by

$$
\begin{aligned}
& \left(Q^{31}\right)_{\alpha \beta}^{11}=\frac{\mathrm{j} k_{\mathbf{s}}^{2}}{\pi} \int_{S}\left[\left(\mathbf{n} \times \boldsymbol{\Phi}_{\beta}^{1}\left(k_{\mathrm{i}} \mathbf{r}\right)\right) \cdot \boldsymbol{\Psi}_{\bar{\alpha}}^{3}\left(k_{\mathbf{s}} \mathbf{r}\right)\right. \\
& \left.+\mathrm{j} m_{\mathrm{r}}\left(\mathbf{n} \times \mathbf{\Psi}_{\beta}^{1}\left(k_{\mathrm{i}} \mathbf{r}\right)\right) \cdot \boldsymbol{\Phi}_{\bar{\alpha}}^{3}\left(k_{\mathbf{s}} \mathbf{r}\right)\right] \mathrm{d} S(\mathbf{r}) \\
& \left(Q^{31}\right)_{\alpha \beta}^{12}=\frac{j k_{\mathbf{s}}^{2}}{\pi} \int_{S}\left[\left(\mathbf{n} \times \boldsymbol{\Psi}_{\beta}^{1}\left(k_{\mathrm{i}} \mathbf{r}\right)\right) \cdot \boldsymbol{\Psi}_{\bar{\alpha}}^{3}\left(k_{\mathbf{s}} \mathbf{r}\right)\right. \\
& \left.+\mathrm{j} m_{\mathbf{r}}\left(\mathbf{n} \times \boldsymbol{\Phi}_{\beta}^{1}\left(k_{\mathrm{i}} \mathbf{r}\right)\right) \cdot \boldsymbol{\Phi}_{\bar{\alpha}}^{3}\left(k_{\mathbf{s}} \mathbf{r}\right)\right] \mathrm{d} S(\mathbf{r}) \\
& \left(Q^{31}\right)_{\alpha \beta}^{21}=\frac{j k_{\mathbf{s}}^{2}}{\pi} \int_{S}\left[\left(\mathbf{n} \times \boldsymbol{\Phi}_{\beta}^{1}\left(k_{\mathrm{i}} \mathbf{r}\right)\right) \cdot \boldsymbol{\Phi}_{\bar{\alpha}}^{3}\left(k_{\mathbf{s}} \mathbf{r}\right)\right. \\
& \left.+\mathrm{j} m_{\mathbf{r}}\left(\mathbf{n} \times \boldsymbol{\Psi}_{\beta}^{1}\left(k_{\mathrm{i}} \mathbf{r}\right)\right) \cdot \boldsymbol{\Psi}_{\bar{\alpha}}^{3}\left(k_{\mathbf{s}} \mathbf{r}\right)\right] \mathrm{d} S(\mathbf{r}) \\
& \left(Q^{31}\right)_{\alpha \beta}^{22}=\frac{j k_{\mathbf{s}}^{2}}{\pi} \int_{S}\left[\left(\mathbf{n} \times \boldsymbol{\Psi}_{\beta}^{1}\left(k_{\mathrm{i}} \mathbf{r}\right)\right) \cdot \boldsymbol{\Phi}_{\bar{\alpha}}^{3}\left(k_{\mathbf{s}} \mathbf{r}\right)\right. \\
& \left.+\mathrm{j} m_{\mathbf{r}}\left(\mathbf{n} \times \boldsymbol{\Phi}_{\beta}^{1}\left(k_{\mathrm{i}} \mathbf{r}\right)\right) \cdot \boldsymbol{\Psi}_{\bar{\alpha}}^{3}\left(k_{\mathbf{s}} \mathbf{r}\right)\right] \mathrm{d} S(\mathbf{r}),
\end{aligned}
$$

with $m_{\mathrm{r}}=\sqrt{\varepsilon_{\mathrm{i}} / \varepsilon_{\mathbf{s}}}$ being the relative refractive index of the particle. The matrix $\mathbf{Q}_{0}^{31}$ has the same structure as the matrix $\mathbf{Q}^{31}$, but it contains as columns the vectors $\mathbf{M}_{\beta}^{1}\left(k_{\mathbf{s}} \mathbf{r}\right)$ and $\mathbf{N}_{\beta}^{1}\left(k_{\mathbf{s}} \mathbf{r}\right)$ in place of the vectors $\boldsymbol{\Phi}_{\beta}^{1}\left(k_{\mathrm{i}} \mathbf{r}\right)$ and $\boldsymbol{\Psi}_{\beta}^{1}\left(k_{\mathrm{i}} \mathbf{r}\right)$, respectively. The expansion coefficients of the scattered field

$$
\mathbf{E}_{\mathbf{s}}(\mathbf{r})=\sum_{\alpha=1}^{N} a_{\mathbf{s} \alpha} \mathbf{M}_{\alpha}^{3}\left(k_{\mathbf{s}} \mathbf{r}\right)+b_{\mathbf{s} \alpha} \mathbf{N}_{\alpha}^{3}\left(k_{\mathbf{s}} \mathbf{r}\right)
$$

are obtained from Huygens principle (or Stratton-Chu representation theorem) by using approximation (7) for the internal surface fields $\mathbf{e}_{\boldsymbol{i}}$ and $\mathbf{h}_{\mathbf{i}}$. We obtain

$$
\left[\begin{array}{c}
a_{\mathbf{s} \alpha} \\
b_{\mathbf{s} \alpha}
\end{array}\right]=\mathbf{Q}^{11}\left(k_{\mathbf{s}}, k_{\mathbf{i}}\right)\left[\begin{array}{c}
a_{\mathbf{i} \beta} \\
b_{\mathbf{i} \beta}
\end{array}\right],
$$

where the matrix $\mathbf{Q}^{11}$ has the same structure as the matrix $\mathbf{Q}^{31}$, but it contains as rows the vectors $\mathbf{M}_{\bar{\alpha}}^{1}\left(k_{\mathbf{s}} \mathbf{r}\right)$ and $\mathbf{N}_{\bar{\alpha}}^{1}\left(k_{\mathbf{s}} \mathbf{r}\right)$ in place of the vectors $\boldsymbol{\Phi}_{\bar{\alpha}}^{3}\left(k_{\mathbf{s}} \mathbf{r}\right)$ and $\boldsymbol{\Psi}_{\bar{\alpha}}^{3}\left(k_{\mathbf{s}} \mathbf{r}\right)$, respectively. Combining (11) and (15) we find that the transition matrix $\mathbf{T}$, relating the scattered field coefficients to the incident field coefficients, is given by

$$
\mathbf{T}=-\mathbf{Q}^{11}\left(k_{\mathbf{s}}, k_{\mathrm{i}}\right)\left(\mathbf{Q}^{31}\left(k_{\mathbf{s}}, k_{\mathrm{i}}\right)\right)^{-1} \mathbf{Q}_{0}^{31}\left(k_{\mathbf{s}}, k_{\mathbf{s}}\right) .
$$

For localized SVWF, we have $\mathbf{Q}_{0}^{31}=\mathbf{I}$, and we are led to the standard representation of the transition matrix.

\section{Algorithm details}

For axisymmetric particles, the scattering problem decouples over the azimuthal modes $m$, and for each $m$, the truncated system of SVWF $\left\{\boldsymbol{\Phi}_{m n}^{q}(k \mathbf{r}), \Psi_{m n}^{q}(k \mathbf{r})\right\}$ reads as 


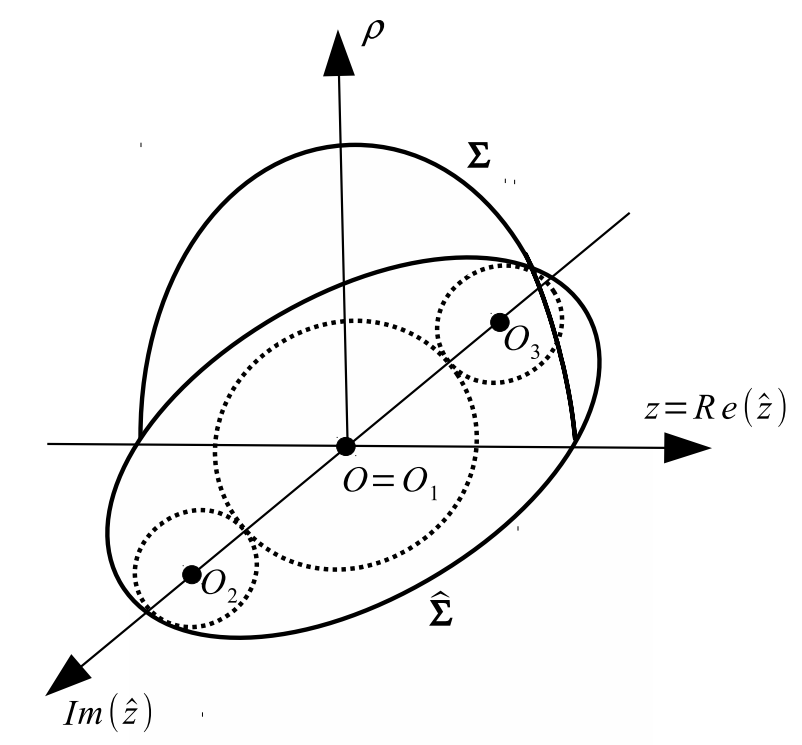

Figure 1: Ilustration of the complex plane. $\Sigma$ is the generatrix of an oblate spheroid, $z$ is the axis of symmetry, $\rho$ is the equatorial axis, and $\widehat{\Sigma}$ is the image of $\Sigma$ in the complex plane. Three poles are chosen along the imaginary axis at positions $\mathrm{O}_{1}, \mathrm{O}_{2}$, and $\mathrm{O}_{3}$. The dotted circles represent the area of influence of each pole.

1. localized SVWF:

$$
\left\{\mathbf{M}_{m n}^{q}(k \mathbf{r}), \mathbf{N}_{m n}^{q}(k \mathbf{r}) \mid n=\max (1,|m|), \ldots, N_{\text {rank }}\right\},
$$

where $N_{\text {rank }}$ is the order of the localized pole,

2. distributed SVWF:

$$
\left\{\mathbf{M}_{m,|m|+l}^{q}\left(k\left(\mathbf{r}-z_{n} \mathbf{e}_{\mathbf{z}}\right)\right), \mathbf{N}_{m,|m|+l}^{q}\left(k\left(\mathbf{r}-z_{n} \mathbf{e}_{\mathbf{z}}\right)\right) \mid n=1, \ldots, N_{\text {rank }}\right\},
$$

where $N_{\text {rank }}$ is number of lowest-order multipoles (discrete sources),

3. multiple SVWF:

$$
\begin{aligned}
& \left\{\mathbf{M}_{m n}^{q}\left(k\left(\mathbf{r}-z_{p} \mathbf{e}_{\mathbf{z}}\right)\right), \mathbf{N}_{m n}^{q}\left(k\left(\mathbf{r}-z_{p} \mathbf{e}_{\mathbf{z}}\right)\right) \mid p=1, \ldots, N_{\mathrm{p}},\right. \\
& \left.n=\max (1,|m|), \ldots, N_{\text {rank } p}\right\},
\end{aligned}
$$

where $N_{\text {rank } p}$ is the order of the pole $p$, and $N_{\text {rank }}=\sum_{p} N_{\text {rank } p}$ is the total expansion order.

In the case of prolate particles, the distribution of the poles on the $z$-axis (axis of symmetry) adequately describes the particle geometry. However, this arrangement is not suitable for oblate particles, in which case, the procedure of analytic continuation of the spherical vector wave functions onto the complex plane along the source coordinate $\widehat{z}$ must be considered. The complex plane $(\operatorname{Re} \widehat{z}, \operatorname{Im} \widehat{z})$ with $\operatorname{Re} \widehat{z}, \operatorname{Im} \widehat{z} \in \mathbb{R}$, is the dual of the azimuthal plane $\varphi=$ const, i.e., $(\rho, z)$ with $\rho \geq 0$ and $z \in \mathbb{R}$, and is defined by taking the real axis $\operatorname{Re} \widehat{z}$ along the $z$-axis. In Fig. 1 we illustrate the complex plane and the curve $\widehat{\Sigma}$, which is the image of the generatrix $\Sigma$ in the complex plane. The programming effort for computing the spherical vector wave functions with an origin located in the complex plane is not very high, because the recurrence relations for the angular functions of complex argument are the same as the recurrence relations for the angular functions of real argument.

For spheroids and cylinders, with which we will deal in our numerical simulations, the poles are chosen as follows: 
1. In the discrete sources method, only lowest-order multipoles are considered, and the poles for scattered and internal field representations have different positions. For oblate particles, the internal poles are distributed on the imaginary axis in the interior and exterior of $\widehat{\Sigma}$, while the external poles are distributed on both the real and imaginary axis in the interior of $\widehat{\Sigma}$. The positions of the poles are generated automatically by using a selection criterion based on the size parameter, particle eccentricity and refractive index.

2. In the null-field method, the poles are placed in the interior of $\widehat{\Sigma}$. The positions of the poles specifying the radiating $\left\{\boldsymbol{\Phi}_{\bar{\alpha}}^{3}\left(k_{\mathbf{s}} \mathbf{r}\right), \boldsymbol{\Psi}_{\bar{\alpha}}^{3}\left(k_{\mathbf{s}} \mathbf{r}\right)\right\}$ and the regular $\left\{\boldsymbol{\Phi}_{\beta}^{1}\left(k_{\mathrm{i}} \mathbf{r}\right), \boldsymbol{\Psi}_{\beta}^{1}\left(k_{\mathrm{i}} \mathbf{r}\right)\right\}$ system of vector functions in (13) are the same. The number of lowest-order multipoles is chosen as an odd number, and the poles are distributed uniformly and symmetrically with respect to the origin. The same rule apply to multiple SVWF excepting the uniform distribution of the poles. In this case, the pole placed at the origin has the largest order, and it is called the dominant pole. The arrangement of other poles is specified by the user. For prolate particles, the poles are distributed along the real axis, while for oblate particles, the poles are distributed along the imaginary axis.

The numerical stability of the methods for solving the underlying system of equations strongly depends on the structure of the matrix to be inverted.

1. In the discrete sources method, the least squares problem

$$
\mathbf{x}=\operatorname{argmin}_{\mathbf{y}}\|\mathbf{A y}-\mathbf{b}\|^{2}
$$

is regularized by means of Tikhonov regularization, i.e.,

$$
\mathbf{x}_{\alpha}=\operatorname{argmin}_{\mathbf{y}}\left(\|\mathbf{A y}-\mathbf{b}\|^{2}+\alpha\|\mathbf{y}\|^{2}\right),
$$

and solved by using the $Q R$ factorization. The regularization parameter $\alpha$ is chosen according to an a priori selection rule based on the size parameter and particle eccentricity.

2. In the null-field method, (1) the Gauss elimination method with backsubstitution [10], and (2) the block matrix inversion method [11] are used. In addition, for large oblate particles, the Gauss elimination routine has been modified to work in multiple-precision arithmetic with the Multiprecision System (MPFUN90) package written by Bailey [28], and parallelized with OpenMP API.

The computations are performed using extended- instead of double-precision floating-point variables. As shown in [20] this is an effective approach for suppressing the numerical instability in computations for large axisymmetric particles with extreme geometries.

For a further improvement of the numerical stability of the null-field method with localized SVWF, the computation of the $\mathbf{T}$ matrix including inversion is performed with multiple-precision arithmetic. The working precision level of the MPFUN90 package is setted to 250 digits.

\section{Convergence analysis}

The convergence procedure of the null-field method checks whether the (total) expansion order, the number of quadrature points, and the number of azimuthal modes are sufficiently large that the scattering characteristics are computed with the desired accuracy. Because the convergence over the azimuthal modes and the number of quadrature points are not problematic, we focus on the convergence over the expansion order, and assume that the incident direction is along the axis of symmetry of the particle. Essentially, the convergence procedure solves the scattering problem for a reference and a lower-order system of SVWF, and checks the convergence of the differential scattering cross-sections at a number of scattering angles [7]. If the calculated results converge within a prescribed tolerance at $80 \%$ of the scattering angles, then convergence is achieved. The choice of the two systems of SVWF is not at all obvious. The method with localized SVWF uses a well ordered set of functions, and in this case, the two systems of SVWF are characterized by $N_{\text {rank }}$ and $N_{\text {rank }}-1$, where is $N_{\text {rank }}$ is the order of the localized pole. For several poles, this choice is not unique because we may change both the position and the expansion order of the poles. In our analysis we use the following selection rules: 
1. For distributed SVWF, the lower-order system is the reference system in which the pole placed at the origin is omitted.

2. For multiple SVWF, the lower-order system is the reference system in which the order of the dominant pole is reduced from $N_{\text {rank1 }}$ to $N_{\text {rank1 }}-1$.

In contrast to the above convergence criterion, which requires the solution of two scattering problems, the discrete sources method uses an "internal convergence criterion", which consists in the estimation of the residual field at the particle surface for a given configuration of poles. Note that close to an edge, where the field can have a singularity, high errors and poor convergence can be obtained. Therefore, it is often preferable to smoothen the edges, for example, with small arcs or with spline approximations of the boundary. Also note that in the framework of the null-field method, a possible method for testing the accuracy of the results for a given configuration of poles is to check the reciprocity condition as discussed by Rother and Wauer [29].

The particles considered in our analysis are spheroids and cylinders. For spheroids, we denote by $a$ the polar radius and by $b$ the equatorial radius, while for cylinders, we denote by $a$ the half-length and by $b$ the radius. The aspect ratio is defined as $a / b$. The discrete sources method has been applied only for spheroids, because the method mainly deals with smooth particle surfaces.

In Fig. 2 we plot the normalized differential scattering cross-sections for a prolate spheroid and a prolate cylinder with a size parameter of $k_{\mathrm{s}} a=80$ and an aspect ratio of $8 / 1$. In these simulations, the null-field method with multiple SVWF is run with $N_{\mathrm{p}}=31, N_{\text {rank }}=101, N_{\text {rank1 }}=11$ and $N_{\text {rank } p}=3$ for $p \neq 1$ in the case of the prolate spheroid, and $N_{\mathrm{p}}=31, N_{\text {rank }}=131, N_{\text {rank1 }}=11$ and $N_{\text {rank } p}=4$ for $p \neq 1$ in the case of the prolate cylinder. The null-field method with distributed SVWF is run with $N_{\text {rank }}=101$ in the case of the prolate spheroid, and $N_{\text {rank }}=131$ in the case of the prolate cylinder. Here, and in the following simulations, the number of integration points is 1000. As demonstrated in Fig. 2, the discrete sources method and the null-field method with discrete sources give the same results. In fact, both methods work excellently for large and highly aspherical prolate particles when the discrete sources are distributed on the axis of symmetry of the particle.

For oblate spheroids, a refractive index of $m_{\mathrm{r}}=1.5+0.02 \mathrm{j}$, and an aspect ratio of $a / b=1 / 4$, the extended-precision version of the null-field method with localized SVWF fails to converge for size parameters $k_{\mathrm{s}} b$ larger than 34 [20]. The same happens with the null-field method with distributed SVWF, but not with the null-field method with multiple SVWF. The reason is that the last method yields a matrix $\mathbf{Q}^{31}$ with the smallest condition number. For example, in the case $k_{\mathrm{s}} b=40$ and $N_{\mathrm{rank}}=80$, the condition number of the matrix $\mathbf{Q}^{31}$ is $4.3 \cdot 10^{18}$ for localized SVWF, $2.5 \cdot 10^{10}$ for distributed SVWF, and $1.3 \cdot 10^{9}$ for multiple SVWF.

In Fig. 3 we plot the normalized differential scattering cross-sections for an oblate spheroid and an oblate cylinder with a size parameter of $k_{\mathrm{s}} b=50$ and an aspect ratio of $1 / 5$. The results are computed with the discrete sources method, the null-field method with multiple SVWF, and the multiple-precision version of the null-field method with localized SVWF. The parameters of calculation of the null-field method with multiple SVWF are $N_{\mathrm{p}}=3, N_{\text {rank }}=96, N_{\text {rank1 }}=60, N_{\text {rank2 }}=N_{\text {rank3 }}=18, k_{\mathrm{s}} \operatorname{Im} z_{1}=0.0$, and $k_{\mathrm{s}} \operatorname{Im} z_{2,3}= \pm 42$ for the oblate spheroid, and $N_{\mathrm{p}}=3, N_{\text {rank }}=130, N_{\text {rank1 }}=90, N_{\text {rank2 }}=N_{\text {rank3 }}=20, k_{\mathrm{s}} \operatorname{Im} z_{1}=0.0$, and $k_{\mathrm{s}} \operatorname{Im} z_{2,3}= \pm 45$ for the oblate cylinder. The reasons for which the discrete sources method still yields convergent results are an appropriate distribution of external poles on the real and imaginary axis, and an ingenious method for smoothing the singularities of the discrete sources. In Table 1 we list the computational time for these simulations. The fastest method is the null-field method with multiple SVWF equipped with the block matrix inversion routine working in extended precision. From Table 1 it is also obvious that the use of the multiple-precison Gauss elimination routine increases the computational time by a factor of 2 , and that the multiple-precision version of the null-field method with localized SVWF is extremely inefficient in terms of computational speed.

The results in Fig. 4 correspond to an oblate spheroid with a size parameter of $k_{\mathrm{s}} b=80$ and an aspect ratio of $1 / 8$, and to an oblate cylinder with a size parameter of $k_{\mathrm{s}} b=70$ and an aspect ratio of $1 / 7$. For these simulations only the null-field method with multiple SVWF converges. Note that our multiple-precision implementation of the null-field method with localized SVWF diverges for aspect ratios smaller than 1/4. 

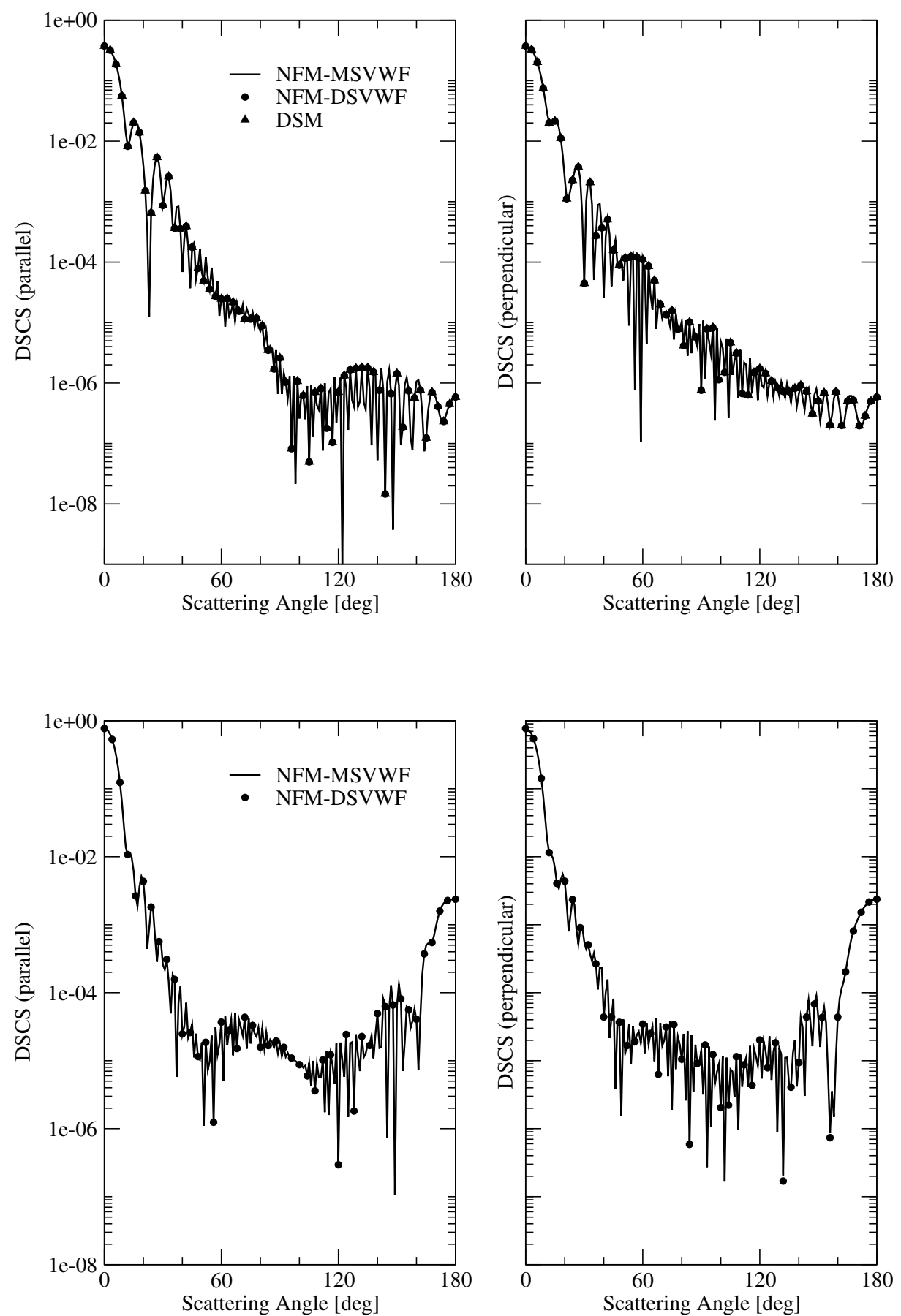

Figure 2: Normalized differential scattering cross-sections for a prolate spheroid (top panels) and a prolate cylinder (bottom panels) with $m_{\mathrm{r}}=1.5+0.02 \mathrm{j}, k_{\mathrm{s}} a=80$ and $k_{\mathrm{s}} b=10$. The plots correspond to the discrete sources method (DSM), and to the null-field method with multiple SVWF (MSVWF), and distributed SVWF (DSVWF). For a clear illustration, only the third points on the DSM and DSVWF curves are shown. 

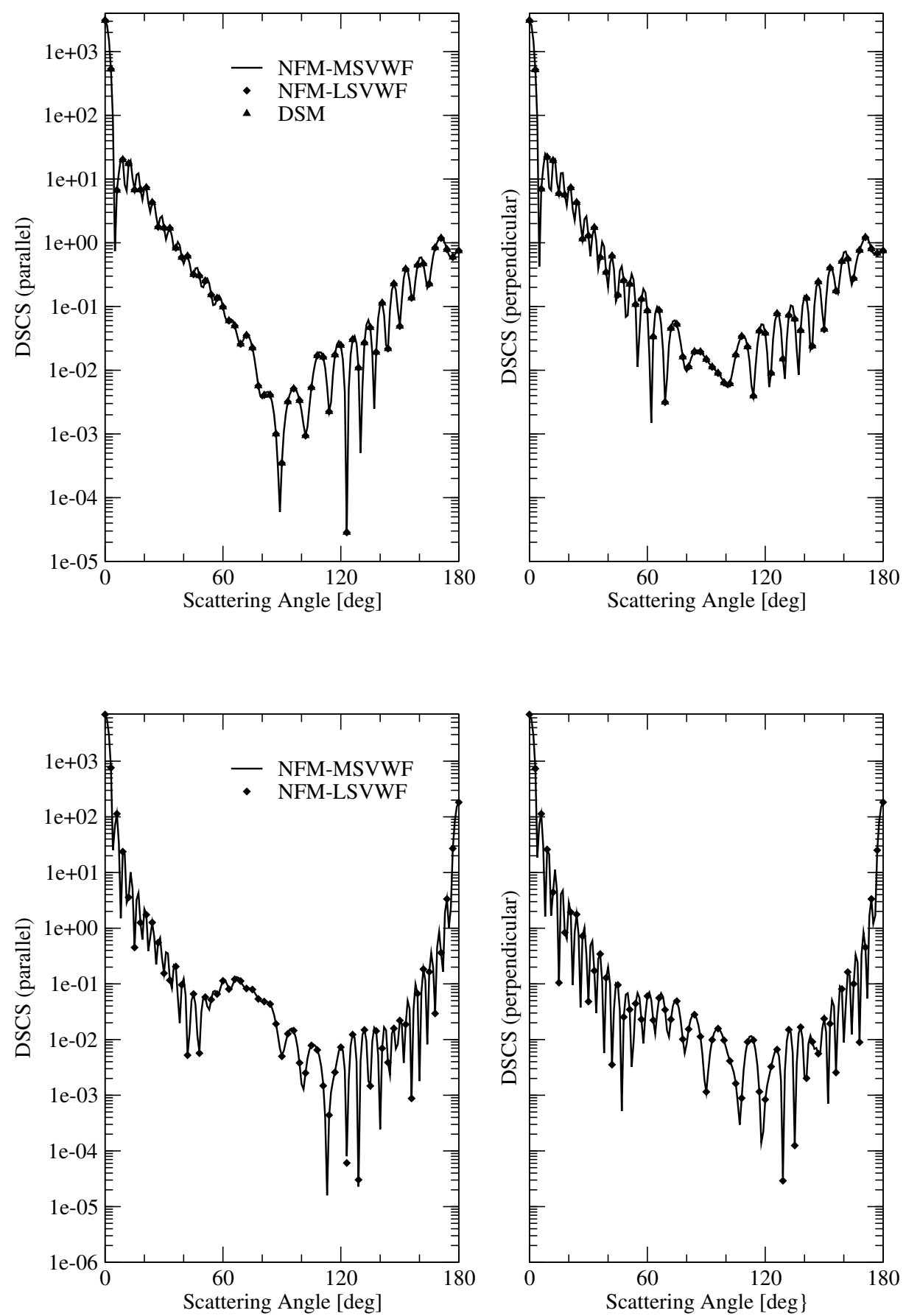

Figure 3: Normalized differential scattering cross-sections for an oblate spheroid (top panels) and an oblate cylinder (bottom panels) with $m_{\mathrm{r}}=1.5+0.02 \mathrm{j}, k_{\mathrm{s}} a=10$ and $k_{\mathrm{s}} b=50$. The plots correspond to the discrete sources method (DSM), and to the null-field method with multiple SVWF (MSVWF), and localized SVWF (LSVWF). For a clear illustration, only the third points on the DSM and LSVWF curves are shown. 


\begin{tabular}{lcc}
\hline & \multicolumn{2}{c}{ Time (min:sec) } \\
\cline { 2 - 3 } Method & Oblate Spheroid & Oblate Cylinder \\
\hline NFM-MSVWF & $3: 42-3: 16-5: 24$ & $6: 20-5: 27-12: 43$ \\
NFM-LSVWF & $58: 33$ & $106: 15$ \\
DSM & $7: 51$ & - \\
\hline
\end{tabular}

Table 1: The computational time for the results in Fig.3. In the case of the null-field method with multiple SVWF, the three values in each column are the computational times corresponding to the Gauss elimination routine working in extended precision, the block matrix inversion routine working in extended precision, and the Gauss elimination routine working in multiple precision.

\begin{tabular}{ccccccccc}
\hline & & & \multicolumn{6}{c}{$N_{\text {rank }}$} \\
\cline { 4 - 9 }$k_{\mathrm{s}} a$ & $N_{\mathrm{p}}$ & $N_{\text {rank }}$ & 0.0 & 55 & 60 & 65 & 70 & 75 \\
\hline 40 & 1 & 120 & 120 & - & - & - & - & - \\
35 & 3 & 152 & 112 & - & - & - & - & 20 \\
30 & 3 & 164 & 112 & - & - & - & - & 26 \\
25 & 3 & 176 & 112 & - & - & - & - & 32 \\
20 & 3 & 196 & 112 & - & - & - & 42 & - \\
15 & 5 & 220 & 112 & - & 42 & - & - & 12 \\
10 & 7 & 264 & 112 & 40 & - & 20 & - & 16 \\
\hline
\end{tabular}

\begin{tabular}{ccccccccc}
\hline & & & \multicolumn{6}{c}{$N_{\text {rank } p}$} \\
\cline { 5 - 9 }$k_{\mathrm{s}} a$ & $N_{\mathrm{p}}$ & $N_{\text {rank }}$ & 0.0 & 45 & 50 & 55 & 60 & 65 \\
\hline 35 & 1 & 114 & 114 & - & - & - & - & - \\
30 & 3 & 138 & 108 & - & - & - & - & 15 \\
25 & 3 & 168 & 108 & - & - & - & 30 & - \\
20 & 5 & 198 & 108 & - & - & 30 & - & 15 \\
15 & 5 & 228 & 108 & - & 40 & - & - & 20 \\
10 & 7 & 268 & 108 & 40 & - & 20 & - & 20 \\
\hline
\end{tabular}

Table 2: The number of poles $N_{\mathrm{p}}$, the total expansion order $N_{\text {rank }}=\sum_{p} N_{\text {rank } p}$, and the expansion order $N_{\text {rank } p}$ of each pole $p$ situated at $\left(0.0, k_{\mathrm{s}} \operatorname{Im} z_{p}\right)$ with $k_{\mathrm{s}} \operatorname{Im} z_{p} \geq 0$, in the complex plane. The poles are placed symmetrically with respect to the origin, while $N_{\mathrm{p}}$ is an odd number. The top table corresponds to oblate spheroids with $m_{\mathrm{r}}=1.5+0.02 \mathrm{j}$ and $k_{\mathrm{s}} b=80$, while the bottom table correspond to oblate cylinders with $m_{\mathrm{r}}=1.311$ and $k_{\mathrm{s}} b=70$.

Because the matrix inversion routines working in extended precision introduce major loss of precision, the Gauss elimination routine working in multiple precision has been used. As a result, the computational times are relatively high: $86 \mathrm{~min}$ for the oblate spheroid and $93 \mathrm{~min}$ for the oblate cylinder. To explain how the null-field method with multiple SVWF works we list in Table 2 the number, expansion order and position of the poles for oblate spheroids of size parameter $k_{\mathrm{s}} b=80$ and oblate cylinders of size parameter $k_{\mathrm{s}} b=70$. In these simulations, the largest dimension $b$ is fixed and the smallest dimension $a$ is decreased, so that the aspect ratio decreases from $1 / 2$ to $1 / 8$ in the case of oblate spheroids, and from $1 / 2$ to $1 / 7$ in the case of oblate cylinders. The case $a / b=1 / 2$ can be handled with a single pole, i.e., by the extended-precision version of the null-field method with localized SVWF. As the aspect ratio $a / b$ is decreased, the total expansion $N_{\text {rank }}$ order is increased by keeping the expansion order of the dominant pole $N_{\text {rank1 }}$ constant and by gradually adding new poles. The poles are introduced by starting from the boundary and by shifting their position toward the origin.

In conclusion, the null-field method with multiple SVWF is superior to the null-field method with distributed SVWF considered so far in [26]. The method can be regarded as an enhancement of the conventional null-field method, because the use of several multipoles leads to better conditioned systems of equations and confers a larger flexibility to the method. 

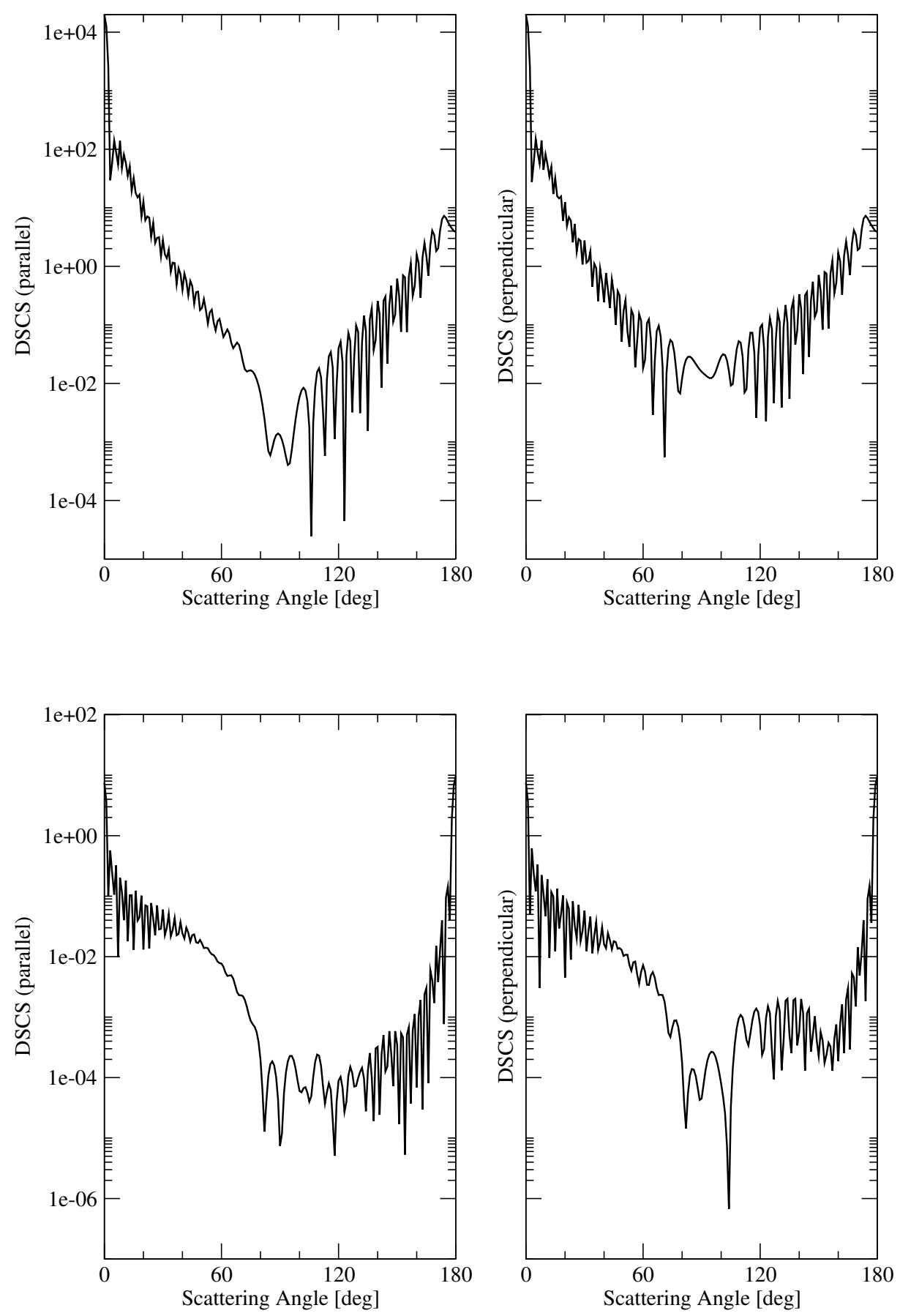

Figure 4: Normalized differential scattering cross-sections for an oblate spheroid with $m_{\mathrm{r}}=1.5+0.02 \mathrm{j}, k_{\mathrm{s}} a=10$ and $k_{\mathrm{s}} b=80$ (top panels) and an oblate cylinder with $m_{\mathrm{r}}=1.311, k_{\mathrm{s}} a=10$ and $k_{\mathrm{s}} b=70$ (bottom panels). The plots correspond to the null-field method with multiple SVWF. 


\section{Conclusions}

The applicability of the discrete sources method with distributed SVWF and the null-field method with localized, distributed and multiple SVWF to electromagnetic scattering by large axisymmetric particles with extreme geometries has been analyzed. The conclusions of our analysis can be summarized as follows:

1. For prolate particles, a distribution of the discrete sources on the axis of symmetry of the particle guarantees the convergence of the computational schemes.

2. For oblate particles, the procedure of analytic continuation of the spherical vector wave functions onto the complex plane has to be considered. In the case of the discrete sources method, convergence is achieved by an appropriate selection of the poles: the external poles are distributed on both the real and imaginary axis, while the internal poles are distributed on the imaginary axis in the interior and exterior of the image of the particle generatrix in the complex plane. In the case of the null-field method, the use of multiple SVWF with poles located on the imaginary axis preserves the decoupling of the scattering problem over the azimuthal modes, and increases the stability of the computational scheme.

For large particles with extreme geometries, the null-field method with multiple SVWF is superior to the null-field method with distributed SVWF and the conventional null-field method. The reason for this enhancement is that the method leads to better conditioned systems of equations. The method is even superior to the conventional null-field method working fully in multiple precision. Unfortunately, an inconvenience of the null-field method with multiple SVWF is the multiple-precision implementation of the inversion algorithm, which increases the computational time by a factor of 2 as compared to an extended-precision implementation. The applicability of matrix inversion algorithms using floating-point format and multiplicative corrections [30] and of the (regularized) total least squares method [31] dealing with errors in the matrix $\mathbf{Q}^{31}$ will be analyzed in the future.

\section{Acknowledgments}

This paper has been elaborated during a two-months visit of Yuri Eremin at the German Aerospace Center in Oberpfaffenhofen. Financial support for this research was provided by DLR-DAAD Research Fellowship Programme No. 154. 
[1] Doicu A, Eremin Y, Wriedt T. Acoustic and electromagnetic scattering analysis using discrete sources. London: Academic Press; 2000.

[2] Eremin YA, Sveshnikov AG. The discrete sources method in electromagnetic diffraction problems. Moscow: Moscow State University Press; 1992.

[3] Hafner C. The generalized multipole technique for computational electromagnetics. Boston, London: Artech House; 1990.

[4] Eremin YA. Representation of fields in terms of sources in the complex plane in the method of non-orthogonal series. Sov Phys Dokl 1983;28:451-452.

[5] Waterman PC. Matrix formulation of electromagnetic scattering. Proc IEEE 1965;53:805-812.

[6] Waterman PC. Symmetry, unitarity and geometry in electromagnetic scattering. Phys Rev D 1971;3:825-839.

[7] Barber PW, Hill SC. Light scattering by particles: computational methods. Singapore: Worl Scientific; 1990.

[8] Mishchenko MI, Travis LD, Lacis AA. Scattering, absorption and emission of light by small particles. Cambridge: Cambridge University Press; 2002.

[9] Lakhtakia A, Varadan VK, Varadan VV. Scattering by highly aspherical targets: EBCM coupled with reinforced orthogonalization. Appl Opt 1984;23:3502-3509.

[10] Moroz A. Improvement of Mishchenko's T -matrix code for absorbing particles. Applied Optics 2005;44:3604-3609.

[11] Petrov D, Shkuratov Y, Videen G. Optimized matrix inversion technique for the T-matrix method. Opt Lett 2007;32:11681170.

[12] Kahnert M, Rother T. Modeling optical properties of particles with small-scale surface roughness: combination of group theory with a perturbation approach. Opt Express 2011;19:11138-11151.

[13] Somerville WRC, Auguié B, Le Ru EC. Severe loss of precision in calculations of T-matrix integrals. J Quant Spectrosc Radiat Transfer 2012;113:524-535.

[14] Somerville WRC, Auguié B, Le Ru EC. A new numerically stable implementation of the T-matrix method for electromagnetic scattering by spheroidal particles. J Quant Spectrosc Radiat Transfer 2013;123:153-168.

[15] Petrov D, Synelnyk E, Shkuratov Y, Videen G. The T-matrix technique for calculations of scattering properties of ensembles of randomly oriented particles with different size. J Quant Spectrosc Radiat Transfer 2006;102:85-110.

[16] Petrov D, Videen G, Shkuratov Y, Kaydash M. Analytic T -matrix solution of light scattering from capsule and bi-sphere particles: applications to spore detection. J Quant Spectrosc Radiat Transfer 2007;108:81-105.

[17] Petrov D, Shkuratov Y, Videen G. Sh -matrices method applied to light scattering by finite circular cylinders. J Quant Spectrosc Radiat Transfer 2008;109:1474-1495.

[18] Petrov D, Shkuratov Y, Videen G. Application of Sh -matrix method to lightscattering by prolate and oblate spheroids. J Opt 2010;12(9): 095701, http://dx.doi.org/10.1088/2040-8978/12/9/095701.

[19] Petrov D, Shkuratov Y, Videen G. An analytical approach to electromagnetic wave scattering from particles of arbitrary shapes. J Quant Spectrosc Radiat Transfer 2011;112(11):1636-1645.

[20] Mishchenko MI, Travis LD. T-matrix computations of light scattering by large spheroidal particles. Opt Commun 1994;109:16-21.

[21] Iskander MF, Lakhtakia A, Durney CH. A new procedure for improving the solution stability and extending the frequency range of the EBCM. IEEE Trans Antennas Propagat 1983;31:317-324.

[22] Lakhtakia A, Varadan VK, Varadan VV. Iterative extended boundary condition method for scattering by objects of high aspect ratios. J Opt Soc Am A 1984;76:906-912.

[23] Wall D. Methods of overcoming numerical instabilities associated with the T-matrix method. In Acoustic, electromagnetic and elastic waves scattering-focus on the T-matrix approach ( Varadan VV, Varadan VK, eds.). New York: Pergamon Press; 1980:269-286.

[24] Bates RHT, Wall DJN. Null field approach to scalar diffraction: I. General method; II. Approximate methods; III. Inverse methods. Phil Trans Roy Soc London 1977;287:45-117.

[25] Hackman RH. The transition matrix for acoustic and elastic wave scattering in prolate spheroidal coordinates. J Acoust Soc Am 1984;75:35-45.

[26] Doicu A, Wriedt T, Eremin Y. Light scattering by systems of particles. Berlin, Heidelberg, New York: Springer; 2006.

[27] Hellmers J, Wriedt T, Doicu A. (2006) Light scattering simulation by oblate disc spheres using the null field method with discrete sources located in the complex plane. Journal of Modern Optics 2006;53:267-282.

[28] Bailey DH. A Fortran 90-Based Multiprecision System. ACM Transactions on Mathematical Software 1995;21:379-387.

[29] Rother T, Wauer J. Case study about the accuracy behavior of three different T-matrix methods. Appl Opt 2010;49:5746-5756.

[30] Rump SM. Inversion of extremely ill-conditioned matrices in floating-point. Japan J Indust Appl Math 2009;26:249-277.

[31] Doicu A, Trautmann T, Schreier F. Numerical regularization for atmospheric inverse problems. Heidelberg, Dordrecht, London, New York: Springer; 2010. 